\title{
3D Local Derivative Pattern for Hyperspectral Face Recognition
}

\author{
Jie Liang ${ }^{1}$ and Jun Zhou ${ }^{2}$ and Yongsheng $\mathrm{Gao}^{3}$ \\ ${ }^{1}$ Research School of Engineering, College of Engineering and Computer Science \\ Australian National University, Canberra, Australia \\ ${ }^{2}$ School of Information and Communication Technology \\ Griffith University, Nathan, Australia \\ ${ }^{3}$ School of Engineering, Griffith University \\ Nathan, Australia
}

\begin{abstract}
Traditional 2D face recognition has been studied for many years and has achieved huge success. Nonetheless, there is high demand to explore unrevealed information other than structure and texture in spatial domain in the faces. Hyperspectral imaging meets such requirements by providing additional spectrum information on objects, in completion to the traditional spatial features extracted in $2 \mathrm{D}$ images. In this paper, we propose a novel 3D high-order texture pattern descriptor for hyperspectral face recognition, which effectively exploit both spatial and spectral features in hyperspectral images. Based on the local derivative pattern, our method encodes the hyperspectral faces with multi-directional derivatives and binarization function in spatial-spectral space. Then a spatialspectral feature descriptor is generated by applying a 3D histogram on the derivative pattern, which can be used to convert hyperspectral face images into vectorized representations. Compared to traditional face recognition methods, our method is able to describe the distinctive micro-patterns which integrate the potential spatial and spectral information in faces. Experiments on the real hyperspectral face databases prove that our method has outperformed several state-of-theart hyperspectral face recognition approaches.
\end{abstract}

\section{INTRODUCTION}

Although a large number of techniques have been developed for face recognition by introducing new feature extraction and pattern recognition methods [16], [18], [14], existing methods still cannot satisfy the requirements of many real-world applications. This is partly due to the deficiency of only using 2D information in the face recognition practice. To address this problem, one solution is to introduce sensors that can capture additional information other than radiance in the visible spectrum range in $2 \mathrm{D}$ space. Examples include range cameras such as Kinects and 3D scanners to capture three dimensional spatial faces, introducing geometrical information to improve the discrimination of individual faces under different orientation [2], [1], [7]. Another promising approach is to exploit spectrum devices to bring in the spectral responses of faces which are supposed to be invariant to external environment. Such studies includes infrared imaging, hyperspectral imaging, etc. [6], [8], [17].

Compared with traditional grayscale and RGB images, hyperspectral images introduce a new dimension of information in terms of spectral responses of objects. Such spectral information is related to the intrinsic material properties of objects, and is measured in continuous narrow bands across wide range of light spectrum. The resulting images usually

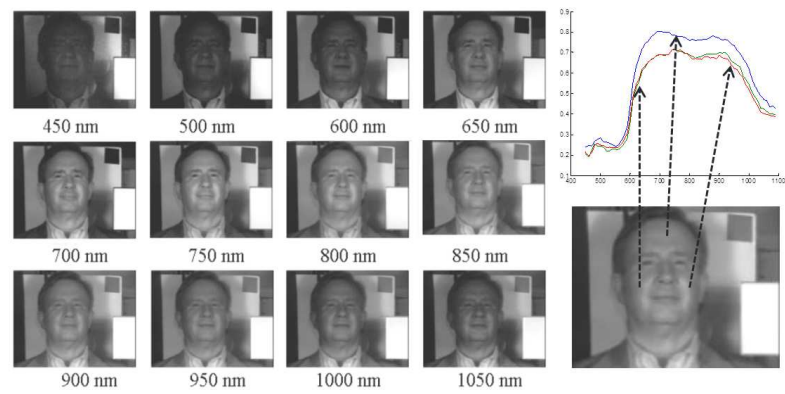

Fig. 1: Hyperspectral face example. On the top right, the spectral responses on the forehead and cheeks are drawn with respect to the wavelength.

consist of tens or hundreds of continuous light wavelength indexed spectral bands, which provide abundant information in both visible and infrared wavelength. An example of hyperspectral face image is shown in Fig. 1 with some band images displayed. This example also shows that the spectral responses on the forehead and cheeks are different for a single subject and the spectral signature is more discriminative than the intensity in single band.

Studies have proved that human skin retains its own spectral properties due to the portion of melanin and hemoglobin [5]. For example, the differences in portion of melanin between African, Asian, and Caucasian lead to obvious distinction in the darkness of skin. Regarding to individuals, this property could be effected by the molecular composition related to tissue, blood and structure. Consequently, spectral responses of the skin has the potential of being an additional discriminative feature for face recognition.

Methods employing both spectral and spatial information for face recognition have been reported in recent years [9], [4], [11], [12]. The challenges for integrating spatial-spectral features are in two aspects. The first one is the low quality of existing hyperspectral face databases [4], [3]. Some spectral bands, which are important for face recognition, are corrupted by heavy noises and have very low signal to noise ratio. Most hyperspectral faces also suffer from the cross-band misalignment resulting from the offset of different wavelength of light, movement of faces and blinking of eyes during the image capturing process which normally takes 
at least several seconds [4], [3]. These factors contaminate the spectral signature that can be extracted from faces. The second and a more serious problem is the totally different intrinsic attributes of spatial and spectral information. Direct fusion of spatial and spectral features can not improve the recognition performance.

Faced with these challenges, researchers have proposed different solutions. Pan et al. pioneered the hyperspectral face recognition by extracting a spectral features picked up from typical areas of faces, such as forehead, cheeks, hair and lips. Experiments proved that their method is robust to the orientation and expression variations [9]. However, this method did not make use of any spatial information. In order to tackle this defect, an extended work integrated the spatial information by introducing a spectral eigenface method [10]. Beside these work, Di et al. performed $(2 D)^{2} \mathrm{PCA}$ on hyperspectral faces and transformed high dimension faces into subspace. A hyperspectral face dataset was collected to analyse this method in whole bands, single band, and band subset with fusion [4]. In the work of Shen and Zheng, a 3D Gabor Wavelet was employed to extract multi-scale and multi-orientation features from the hyperspectral faces [11]. This approach achieved a significant higher accuracy than all fore-mentioned methods on the benchmark dataset in [4]. Despite of its high accuracy, it suffered from huge computation and memory costs. In more recent, Unzir et al. developed a spatial-spectral feature extraction method based on 3D discrete cosine transform (DCT) to calculate low frequency coefficients of hyperspectral face images. By using a partial least square regression model to perform face classification, this method achieved more than $90 \%$ recognition rate on three databases [12]. Regarding to spatial-spectral feature extraction, all these work did not completely solve the two challenges mentioned above. They either only use spectral signature or treat the hyperspectral data as an isotropic volume.

In this paper, we propose a $3 \mathrm{D}$ high-order texture pattern descriptor based on local derivative pattern (LDP) for hyperspectral face recognition. LDP is a high-order derivative descriptor which provides a general framework for encoding directional features on 2D images [13]. It can be represented as a high-order and multi-direction derivative plus a special binarization function which acts as a denoising function. By combining these two functions, LDP is very efficient to encode the detailed information and suppresses the noise at the same time. In this paper, we introduce the $3 \mathrm{D}$ local derivative pattern (3D LDP) to extract the spatial-spectral information from hyperspectral face images. A 3D histogram is constructed on the derivative pattern and then used as the feature descriptor for face recognition. It should be noted that our method is superior to the $3 \mathrm{D}$ extension of local binary pattern (3D LBP) which is originally developed for dynamic texture analysis [15]. It is validated on two benchmark hyperspectral face datasets. The results show that it outperforms several state-of-the-art methods and is robust to time variations.

The rest of the paper is organized as follows. Section II
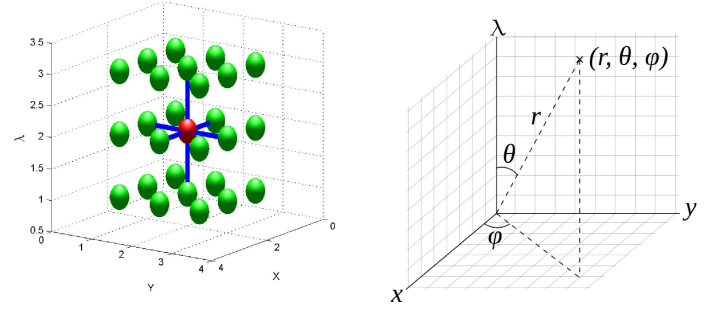

Fig. 2: Coordinate systems in 3D space

describes the proposed 3D LDP method and how the extracted texture feature is converted to a descriptor for each hyperspectral image. The implementation details are described in section III, followed by experiments and analysis in section IV. Finally, the conclusions are drawn in section V.

\section{3D Local Derivative PatTern}

The proposed 3D LDP is a three dimensional high-order texture descriptor. It analyses the micro-patterns in three dimensional data and encode them into binary numbers. The method consists of two parts, a 3D directional derivative pattern and a special binarization function. The 3D directional derivative pattern describes the changes in multidirections, and curvature in high orders. The binarization function provides a general description on the consistency of two derivatives, which is suitable to extract detailed features in multi-dimensional data. Though hyperspectral images provide additional spectral information, there is huge redundancy along the wavelength dimension. It is because that spectral responses of objects change smoothly across most wavelengths and the discriminative spectral information may only exist in several specific wavelengths. Therefore, 3D LDP has the potential to extract the spatial-spectral features in hyperspectral faces. After the 3D LDP features are calculated, they are converted into histogram to generate vectorized image representation.

\section{A. Construction of Local Derivative Pattern}

Given a hyperspectral image represented as a cube, a cartesian coordinate system and a spherical coordinate system can be defined. A $3 \times 3 \times 3$ example is shown in Fig. 2. In the 3D coordinate system, a point $P$ is represented by its index of $(x, y, \lambda)$ while angle/direciton is expressed by the combination of inclination $\theta$ and azimuth $\varphi$. Given the 3D neighborhood of a central point, the first-order 3D directional derivative $I_{(\theta, \varphi)}^{\prime}(x, y, \lambda)$ is defined as follows

$$
\begin{array}{r}
I_{(\theta, \varphi)}^{\prime}(x, y, \lambda)=I(x+\triangle x, y+\triangle y, \lambda+\triangle \lambda)-I(x, y, \lambda) \\
\theta=\arctan \left(\frac{\sqrt{\triangle x^{2}+\triangle y^{2}}}{\triangle \lambda}\right), \varphi=\arctan \left(\frac{\triangle y}{\triangle x}\right)
\end{array}
$$

where $P=(x+\triangle x, y+\triangle y, \lambda+\triangle \lambda)$ is the nearest neighbor of the central point $P=(x, y, \lambda)$ in direction of $(\theta, \varphi)$. It should be noticed that in the discrete 3D image space, $\theta$ and $\varphi$ are also discrete which means $\theta \in$ 
$\{k \pi / 4 \mid k=0,1,2,3\}$ and $\varphi \in\{b \pi / 4 \mid b=0,1, \ldots, 7\}$. Given the directional derivative calculated in a specific angle, higher order derivative can be calculated in the same angle.

In order to encode the derivative, our method employs a function to binarize the derivative between the central point and its neighbours. This function describes the consistency of two neighboring derivatives. It is defined as follows

$$
f\left(I^{\prime}\left(P_{c}\right), I^{\prime}\left(P_{i}\right)\right)= \begin{cases}0 & \text { if } I^{\prime}\left(P_{c}\right) * I^{\prime}\left(P_{i}\right)>0 \\ 1 & \text { if } I^{\prime}\left(P_{c}\right) * I^{\prime}\left(P_{i}\right) \leq 0\end{cases}
$$

where $I^{\prime}\left(P_{i}\right)$ is the derivative calculated at the $i^{t h}$ neighbour of the central point $P_{c}$. When the derivatives are consistent, i.e., both are positive or negative, the result is 0 , otherwise, the result is 1 .

The directional derivative and binarization function play different roles and are independent to each other. The former extracts various distinctive spatial and spectral changes, while the latter quantizes the consistency between derivatives of the central point and its neighbours. From this point of view, the binarization function can be considered as another level of derivative on top of the directional derivative of the hyperspectral image, i.e., a special second order derivative of the raw image. Therefore, given point $P_{c}$, we denote such second order derivative as $D_{(\theta, \varphi)}^{2}\left(P_{c}\right)$, such that

$$
D_{(\theta, \varphi)}^{2}\left(P_{c}\right)=f\left(I_{(\theta, \varphi)}^{\prime}\left(P_{c}\right), I_{(\theta, \varphi)}^{\prime}\left(P_{i}\right)\right)
$$

After obtaining the binary derivative, these values are fed into an encoding system to generate a unique integer which encodes the local derivative pattern as follows

$$
3 \operatorname{DLDP}_{(\theta, \varphi)}^{2}=\sum_{i=1}^{m} D_{(\theta, \varphi)}^{2} \times 2^{i-1}
$$

where $i$ indexes the $i^{\text {th }}$ neighbour in the $m$ neighbourhood of the central point.

When all directions are combined, the final second-order 3D LDP becomes

$$
\begin{aligned}
& 3 D L D P^{2}= \\
& \sum_{\theta \in A, \varphi \in B} \sum_{i=1}^{m} f\left(I_{(\theta, \varphi)}^{\prime}\left(P_{c}\right), I_{(\theta, \varphi)}^{\prime}\left(P_{i}\right)\right) \times 2^{(j-1) \times m+i-1}
\end{aligned}
$$

where $A=\{k \pi / 4 \mid k=0,1,2,3\}$ is the set of angles for $\theta$ and $B=\{b \pi / 4 \mid b=0,1, . ., 8\}$ is the set of angles for $\varphi$ in the discrete 3D space in Fig. 4. Different combinations of $\theta$ and $\varphi$ determine different directions for derivative calculation, and $j$ is the index for these combination.

\section{B. $n^{\text {th }}$-order Local Derivative Pattern}

The 3D LDP can be extended to higher-order derivatives. This can be implemented by applying equation (1) iteratively and then perform binarization function. Consequently, the $n^{\text {th }}$-order derivative of 3D LDP is constructed by calculating the directional derivative for $n-1$ times before applying the

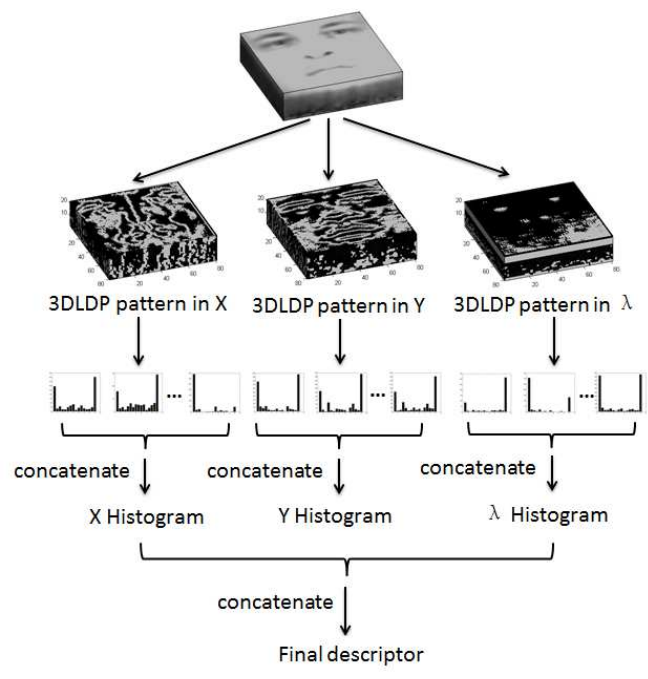

Fig. 3: 3D LDP descriptor construction.

binarization function in Equation (2). Therefore, the $n^{t h}$ order derivative is defined as

$$
D_{(\theta, \varphi)}^{n}\left(P_{c}\right)=f\left(I_{(\theta, \varphi)}^{n-1}\left(P_{c}\right), I_{(\theta, \varphi)}^{n-1}\left(P_{i}\right)\right)
$$

After the encoding step, the $n^{\text {th }}$-order 3D LDP code in the direction of $(\theta, \varphi)$ within the $m$ neighborhood of $P_{c}$ is calculated as

$$
\begin{aligned}
& 3 D L D P_{(\theta, \varphi)}^{n}= \\
& \sum_{i=1}^{m} f\left(I_{(\theta, \varphi)}^{n-1}\left(P_{c}\right), I_{(\theta, \varphi)}^{n-1}\left(P_{i}\right)\right) \times 2^{(j-1) \times m+i-1}
\end{aligned}
$$

The 3D LDP code extracted in different directions can be combined into a final 3D LDP code as follows

$$
\begin{aligned}
& 3 D L D P^{n}= \\
& \sum_{\theta \in A, \varphi \in B} \sum_{j=1}^{m} f\left(I_{(\theta, \varphi)}^{n-1}\left(P_{c}\right), I_{(\theta, \varphi)}^{n-1}\left(P_{i}\right)\right) \times 2^{(j-1) \times m+i-1}
\end{aligned}
$$

where the parameters have the same meaning as in Equation (5).

\section{Construction of $3 D$ LDP Descriptor}

Through the above procedure, each pixel is assigned with an integer at given direction $(\theta, \varphi)$. Such mixed-order derivative code contains discriminative texture feature of a local neighborhood in a hyperspectral image. To convert such texture feature into a vectorized descriptor for face recognition, statistical distribution of the 3D LDP feature in local regions is calculated and summarized using a histogram. The length of each histogram is $2^{m}$ for each direction. In practice, the length of histogram can be shortened by merging the neighboring entries to generate smaller number of bins.

The hyperspectral image is divided into small cubes. On each cube, a local histogram is generated in each direction. For each direction, the local histograms are concatenated, such that the resulting directional histogram embeds both spatial and spectral information at the region level. Then the directional histograms are merged into a final histogram which is the final descriptor of the hyperspectral face image. 

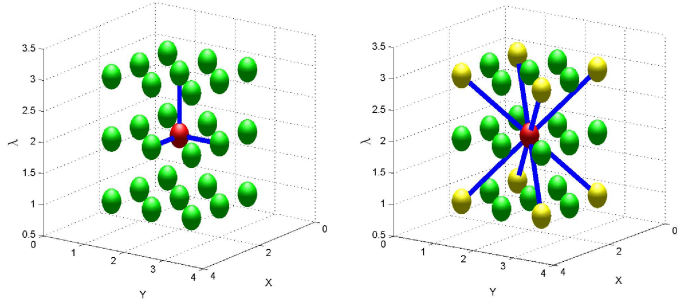

Fig. 4: Left panel: directions of 3D LDP. Right panel: 8 neighbours chosen to code the pattern.

An example of 3D LDP descriptor extracted from hyperspectral face image is displayed in Fig. 3.

\section{Hyperspectral Face Recognition}

A distance measurement between hyperspectral face images is required for the final recognition step. In our method, histogram intersection is adopted to measure the similarity between two histograms

$$
M_{12}=\sum_{i}^{K} \min \left(H_{1}(i), H_{2}(i)\right)
$$

where $M_{12}$ is the histogram intersection between histogram $H_{1}$ and $H_{2} . K$ is the length of the histogram. This measurement calculates the common area of two histograms. The higher the value is, the more similar two histograms are.

\section{IMPLEMENT DETAILS}

In our method, we first filter the hyperspectral faces with a 3D Gabor filter. It is necessary because hyperspectral images often suffer from serious noises coming from both hardware and the imaging process. Directly applying 3D LDP will result in encoding a lot of noises in the spectral domain. The 3D Gabor reduces the influence of sensor noise and cross-band misalignment. It also enhances the discriminative patterns in the spatial and spectral domain. Different from the 3D Gabor Wavelet method in [11], filters with fixed scale are used.

In a 3D space, a pixel can have up to 26 direct neighboring pixels. In our implement, we make use of 3 directions and 8 neighbours in the cube as shown in Fig.4. These three directions are $(0,0),(\pi / 2,0)$ and $(\pi / 2, \pi / 2)$, which correspond to positive $x$, positive $y$, and positive $\lambda$, respectively. The 8 neighbours are the eight corners of the cube which span both spatial and spectral dimensions. It should be mentioned that this is the most basic form and more directions and neighbours can be used to extract more detailed information. In the implementation, we calculate the second order 3D LDP. A summary of the second-order 3D LDP is given in Algorithm 1. Its extension to higher order is straightforward.

\section{EXPERIMENTS AND RESUlTS}

The experiments consist of two parts. In the first part, we compare the proposed methods with the state-of-theart hyperspectral face recognition algorithms on two wellestablished hyperspectral face datasets. For the completeness

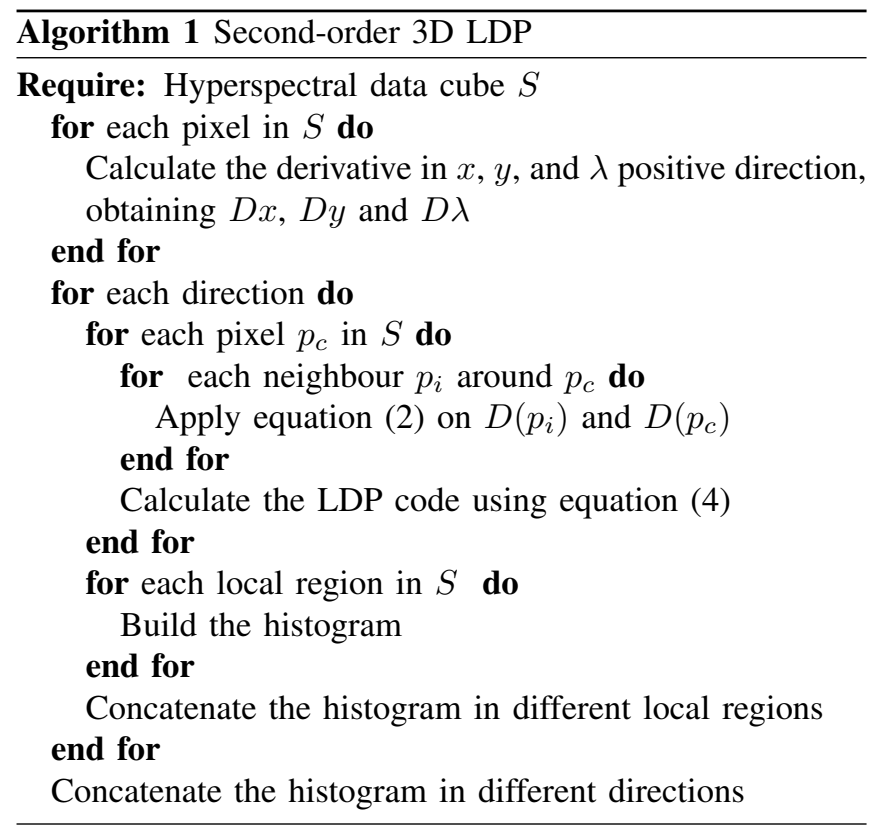

of experiments, we also implement the 3D LBP in our test. The results show that our method outperforms all other approaches. Then in the second part, we validate the usefulness of spectral information by comparing the proposed method with a state-of-the-art 2D face recognition method.

Hyperspectral face recognition is much less mature than work in 2D faces. There are very few datasets available. Furthermore, some hyperspectral face datasets are not publicly available due to privacy or other reasons such as those collected and used in [9] and [12]. To our knowledge, there are only two publicly available datasets of hyperspectral face, i.e., Hong Kong Polytechnic University Hyperspectral Face Database (HK-PolyU) [4] and Carnegie Mellon University Hyperspectral Face Dataset (CMU dataset) [3]. As a consequence, our experiment were conducted only on these two datasets.

\section{A. Results on HK-PolyU Hyperspectral Face Dataset}

The first dataset is the Hong Kong Polytechnic University Hyperspectral Face Database (HK-PolyU) [4], which consists of 300 hyperspectral faces from 25 subjects. For each subject, there were four sessions collected at two time with an average span of five months. Each session consists of frontal, right and left views with the neutral expression. Each image covers the visible wavelength from $400 \mathrm{~nm}$ to $720 \mathrm{~nm}$ with an interval of $10 \mathrm{~nm}$. The images are quite noisy at the both ends of the spectral range. So the first six and last 3 bands are removed in the experiment. Fig. 5 shows examples of two subjects taken in four sessions. It can be seen that the appearance of the same person varies a lot in different sessions. The bands of this database are not well registered and the pixel based spectral signature are contaminated. This explains why it cannot be used directly for 3D LDP.

In order to evaluate the performance of 3D LDP, we compare it with several hyperspectral feature extraction methods. 

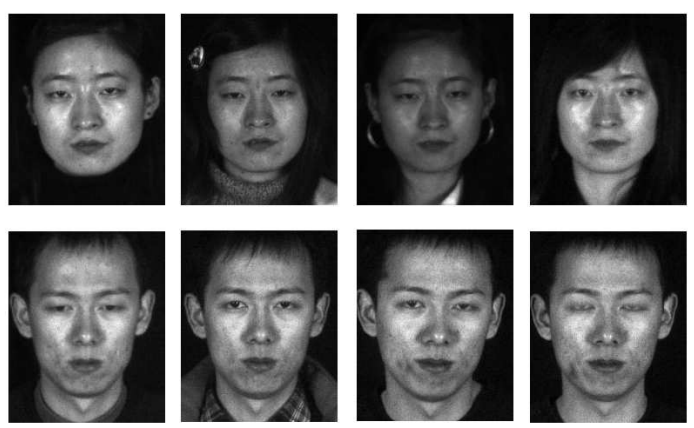

Fig. 5: Examples from the HK-PolyU Hyperspectral Face Database. Images are extracted from different sessions.

These methods are Spectral Feature [9], Spectral Eigenface [10], 2D PCA [4], 3D LBP [15], 3D Gabor Wavelet [11] and a newly developed method of 3D DCT [12]. We followed the experiment setting in [11]. The hyperspectral faces were cropped into $64 \times 64$ in spatial dimension and the position of eyes were manually aligned. Four sessions of 25 subjects were used to as the test set. The gallery set was constructed by randomly sampling two sessions from test set and the rest faces were used as the probe set. For our method, 13 3D Gabor transforms (frequency $=0.25$, orientation $1=0, \pi / 4$, $\pi / 2,3 \pi / 4$, orientation $2=0, \pi / 4, \pi / 2,3 \pi / 4$ ) were used to preprocess the hyperspectral faces. We fixed the subregion size to $8 \times 8 \times 8$ pixels to build the $3 \mathrm{D}$ histogram and set the derivative order to two. The 3D LBP uses the same setting with 3D LBP except that it takes 14 neighbours as introduced in [15]. In 3D Gabor Wavelet, the size of Gabor was $39 \times 39 \times 39$ and 52 filters were used as recommended in [11]. The 3D DCT method reported in [12] consists of a 3D DCT based feature extraction method and a partial least squares regression based classification method. We only implemented the feature extraction part using the code published by the authors of [12] because our paper focuses more on the spatial-spectral feature extraction. The face recognition in alternative methods was implemented by using the nearest neighbor classifier based on the Euclidean distance between probe and gallery images, while our method used the nearest neighbor classifier based on histogram intersection. We used the mean and standard deviation of rank-1 recognition rate of six possible combinations of the gallery and probe sets to evaluate the performance of each method.

TABLE I: Recognition rate of different methods on the HKPolyU Hyperspectral Face Database.

\begin{tabular}{|l|c|}
\hline Methods & Recognition Rate \\
\hline Spectral Feature[9] & $45.35 \pm 3.87 \%$ \\
\hline Spectral Eigenface[10] & $70.33 \pm 3.61 \%$ \\
\hline 2D PCA[4] & $71.00 \pm 3.16 \%$ \\
\hline 3D DCT[12] & $84.00 \pm 3.35 \%$ \\
\hline 3D LBP[15] & $88.80 \pm 1.79 \%$ \\
\hline 3D Gabor Wavelet[11] & $90.00 \pm 2.83 \%$ \\
\hline 3D LDP & $\mathbf{9 5 . 3 3} \pm \mathbf{1 . 6 3 \%}$ \\
\hline
\end{tabular}

The experimental results are shown in Table I. 3D LDP has achieved the highest recognition with $95.33 \pm 1.63 \%$ on the HK-PolyU database, exceeding the second best 3D Gabor Wavelet by $5.33 \%$ in average. The 3D Gabor Wavelet feature extraction method has achieved similar results as reported in [11] and [12]. Just as LDP performs better than LBP in $2 \mathrm{D}$ face recognition, the $3 \mathrm{D}$ version also shows the same trend in which 3D LDP is $6.53 \%$ higher than 3D LBP. The accuracy of 3D DCT is $84.00 \%$, which is much lower than the results reported in [12]. This is partly due to the fact that the partial least square regression has not been used as the classifier. Compared with 3D LDP and 3D Gabor methods, 3D DCT only extracts low-frequency coefficients of DCT, which is a general representation of signal energy. In contrast, 3D LDP and 3D Gabor Wavelet extract multi-scale and multi-direction local textures, which capture more local information than 3D DCT. The results also show that pure spectral feature performs the worst because it has ignored the spatial information.

\section{B. Results on CMU Hyperspectral Face Dataset}

The second hyperspectral face dataset is the Carnegie Mellon University Hyperspectral Face Dataset (CMU dataset) [3]. Examples of this dataset are shown in Fig. 6. Compared with the HK-PolyU dataset, the CMU dataset covers a wider range of spectrum from $450 \mathrm{~nm}$ to $1090 \mathrm{~nm}$ with an interval of $10 \mathrm{~nm}$. It includes data of 54 subjects taken in multiple sessions. Each session consists of four different illumination conditions which include all lights on, center light on, left light on and right light on. Each subject has 1 to 5 sessions taken at different times. Due to the low spectral sensitivity of hyperspectral camera, bands at the two ends of spectrum range have very low signal to noise ratio. In this experiment, faces under the condition of all lights on taken at different time were used as the test set. The gallery set was constructed by randomly sampling one face per subject from the test set and the rest faces were used as the probe set. This procedure repeated 10 times and the mean and standard deviation were used to evaluate the performance of different methods.
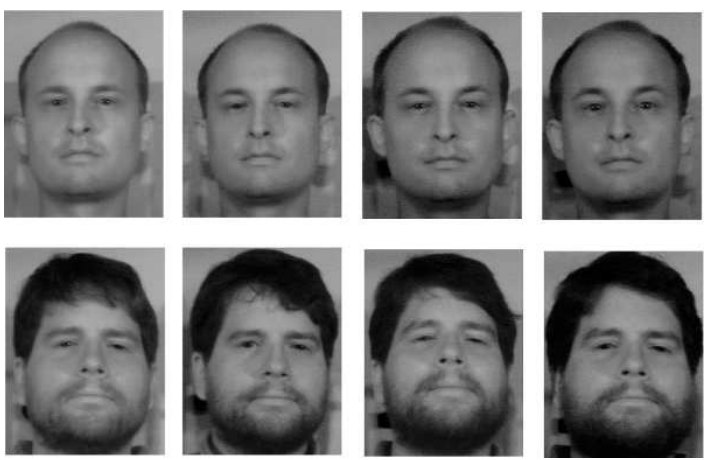

Fig. 6: Example images on two subjects in the CMU Hyperspectral face dataset. For each subject, the images are captured in four sessions with $700 \mathrm{~nm}$ band displayed.

The gallery included 47 faces while the probe had 98 faces. Each face was cropped into $64 \times 64$ in spatial dimension and the position of eyes were manually aligned. The first 
7 and last 2 noisy bands were removed. In this experiment, we adopted the same setting as on the HK-PolyU dataset for 3D LBP, 3D Gabor Wavelet, 3D DCT and 3D LDP.

TABLE II: Recognition rates of different methods on the CMU Hyperspectral Face Dataset.

\begin{tabular}{|l|c|}
\hline Methods & Recognition Rate \\
\hline Spectral Feature[9] & $38.18 \pm 1.89 \%$ \\
\hline 2D PCA[4] & $72.10 \pm 5.41 \%$ \\
\hline Spectral Eigenface[10] & $84.54 \pm 3.78 \%$ \\
\hline 3D DCT[12] & $88.65 \pm 2.34 \%$ \\
\hline 3D LBP[15] & $92.16 \pm 3.52 \%$ \\
\hline 3D Gabor Wavelet[11] & $92.20 \pm 2.46 \%$ \\
\hline 3D LDP & $\mathbf{9 4 . 8 3} \pm \mathbf{2 . 6 2 \%}$ \\
\hline
\end{tabular}

The experimental results are shown in Table II. These results are consistent with those on the HK-PolyU dataset. The 3D LDP leads the performance among all the methods with an average recognition rate of $94.83 \%$. The second and third methods are 3D Gabor Wavelet and 3D LBP. Regarding the efficiency, 3D DCT is the fastest because it only produces one cube per face, while the 3D Gabor Wavelet is the worst which produces 52 cubes. The 3D LBP and 3D LDP are in the middle by creating 13 cubes per face. Overall, the 3D LDP efficiently extracts the spatial-spectral features and performs the best in recognition rate among all methods.

\section{Further Analysis of $3 D L D P$}

In order to show that the spectral information provided by the hyperspectral images increases the accuracy of face recognition, we also conducted an experiment in which 3D LDP was compared with a $2 \mathrm{D}$ face recognition method. We chose 2D LDP for comparison because it uses the same rationale in feature extraction in $2 \mathrm{D}$ space. Because $2 \mathrm{D}$ LDP cannot be directly applied to hyperspectral images, we ran it on each individual band. Then average recognition rate and standard deviation from all bands are calculated. In 2D LDP, the subregion size was set to $8 \times 8$ pixels and the derivative order was set to two. The comparison is conducted on both datasets following the same setting as experiment $\mathrm{A}$ and $\mathrm{B}$. The results in Table III show that 3D LDP performs significantly better than 2D LDP on both datasets. This proves the value of introducing spectral information for face recognition. Effective characterizing the spatial-spectral information has led to remarkable benefit in improving the accuracy of face recognition.

TABLE III: Recognition rate of 3D LDP and 2D LDP on two datasets.

\begin{tabular}{|l|c|c|}
\hline Methods & HKPolyU database & CMU database \\
\hline 2D LDP [13] & $86.25 \pm 9.62 \%$ & $90.27 \pm 3.51 \%$ \\
\hline 3D LDP & $95.33 \pm 1.63 \%$ & $94.83 \pm 2.62 \%$ \\
\hline
\end{tabular}

\section{CONCLUSION}

In this paper, we have introduced a 3D texture descriptor for hyperspectral face recognition. It provides a framework for constructing a multi-direction and multi-neighbourhood local derivative patterns. This pattern efficiently integrates the information from both spatial and spectral domain and reduces the negative influence of noise in the hyperspectral images. By building a 3D histogram on this feature, a spatialspectral descriptor can be generated for hyperspectral face recognition. The proposed method has been tested on two public hyperspectral face datasets and has been compared with several existing methods. The results prove that our method has outperformed the state-of-the-art methods in terms of both accuracy and efficiency. It is expected that the proposed method can also be used in other hyperspectral classification applications.

\section{REFERENCES}

[1] K. W. Bowyer, K. Chang, and P. Flynn. A survey of approaches and challenges in $3 \mathrm{D}$ and multi-modal $3 \mathrm{D}+2 \mathrm{D}$ face recognition. Computer Vision and Image Understanding, 101(1):1-15, 2006.

[2] A. M. Bronstein, M. M. Bronstein, and R. Kimmel. Expressioninvariant 3D face recognition. In Proceedings of the International Conference on Audio- and Video-based Biometric Person Authentication, pages 62-70, 2003.

[3] L. J. Denes, P. Metes, and Y. Liu. Hyperspectral face database. Technical Report CMU-RI-TR-02-25, Robotics Institute, 2002.

[4] W. Di, D. Zhang, D. Zhang, and Q. Pan. Studies on hyperspectral face recognition in visible spectrum with feature band selection. IEEE Transactions on Systems, Man and Cybernetics, Part A: Systems and Humans, 40(6):1354-1361, 2010.

[5] T. Igarashi, K. Nishino, and S. K. Nayar. The appearance of human skin: A survey. Foundations and Trends in Computer Graphics and Vision, 3(1):1-95, 2007.

[6] S. G. Kong, J. Heo, B. R. Abidi, J. Paik, and M. A. Abidi. Recent advances in visual and infrared face recognition - a review. Computer Vision and Image Understanding, 97:103-135, 2005.

[7] B. Li, A. Mian, W. Liu, and A. Krishna. Using Kinect for face recognition under varying poses, expressions, illumination and disguise. In IEEE Workshop on Applications of Computer Vision, pages 186-192, 2013.

[8] S. Li, R. Chu, S. Liao, and L. Zhang. Illumination invariant face recognition using near-infrared images. IEEE Transactions on Pattern Analysis and Machine Intelligence, 29(4):627-639, 2007.

[9] Z. Pan, G. Healey, M. Prasad, and B. Tromberg. Face recognition in hyperspectral images. IEEE Transactions on Pattern Analysis and Machine Intelligence, 25(12):1552-1560, 2003.

[10] Z. Pan, G. Healey, and B. Tromberg. Comparison of spectral-only and spectral/spatial face recognition for personal identity verification. EURASIP Journal on Advances in Signal Processing, pages 8:1-8:6, 2009.

[11] L. Shen and S. Zheng. Hyperspectral face recognition using 3D gabor wavelets. In Proceedings of the International Conference on Pattern Recognition, pages 1574-1577, 2012.

[12] M. Uzair, A. Mahmood, and A. Mian. Hyperspectral face recognition using 3D-DCT and partial least squares. In Proceedings of the British Machine Vision Conference, pages 57.1-57.10, 2013.

[13] B. Zhang, Y. Gao, S. Zhao, and J. Liu. Local derivative pattern versus local binary pattern: Face recognition with high-order local pattern descriptor. IEEE Transactions on Image Processing, 19(2):533-544, 2010.

[14] X. Zhang and Y. Gao. Face recognition across pose: a review. Pattern Recognition, 42(11):2876 - 2896, 2009.

[15] G. Zhao and M. Pietikainen. Dynamic texture recognition using local binary patterns with an application to facial expressions. IEEE Transactions on Pattern Analysis and Machine Intelligence, 29(6):915-928, June 2007.

[16] W. Zhao, R. Chellappa, P. Phillips, and A. Rosenfeld. Face recognition: A literature survey. ACM Computing Surveys, 35(4):399-458, 2003.

[17] Y. Zheng and A. Elmaghraby. A brief survey on multispectral face recognition and multimodal score fusion. In IEEE International Symposium on Signal Processing and Information Technology, pages 543-550, 2011.

[18] X. Zou, J. Kittler, and K. Messer. Illumination invariant face recognition: A survey. In IEEE International Conference on Biometrics: Theory, Applications, and Systems, pages 1-8, 2007. 